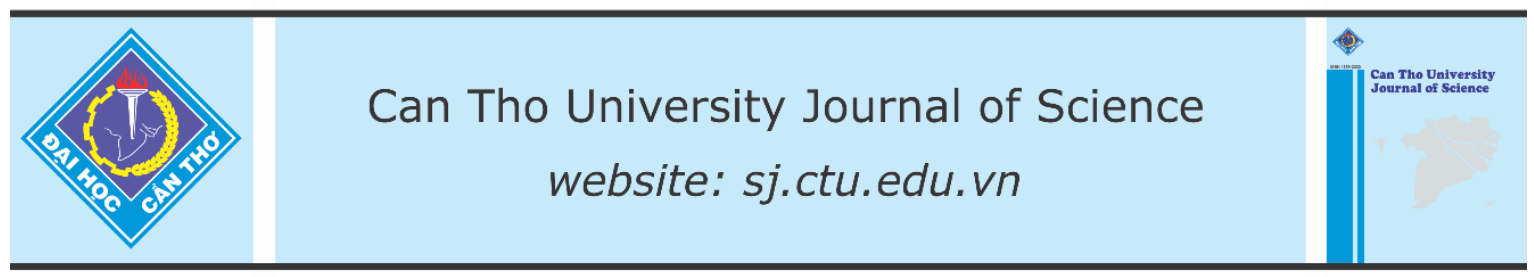

DOI: 10.22144/ctu.jen.2021.010

\title{
Bimetallic CuCo-Zeolitic imidazole frameworks (CuCo-ZIFs): Synthesis and characterization
}

Dang Huynh Giao ${ }^{1 *}$, Truong Tuong Vi ${ }^{1}$, Ho Thi Thu Ngan ${ }^{1}$, Nguyen Van Binh ${ }^{1}$, Ho Ngoc Tri Tan ${ }^{1}$ and Pham Van Toan ${ }^{2}$

${ }^{1}$ College of Engineering Technology, Can Tho University

${ }^{2}$ College of Environment and Natural Resources, Can Tho University

*Correspondence: Dang Huynh Giao (email: dhgiao@ctu.edu.vn)

\section{Article info.}

Received 04 Oct 2020

Revised 28 Oct 2020

Accepted 31 Mar 2021

\section{Keywords}

Bimetallic, CuCo-ZIFs, ultrasound, zeolitic imidazole frameworks

\begin{abstract}
A kind of bimetallic Cu/Co zeolitic imidazole frameworks ( $\mathrm{CuCo}$-ZIFs) has been successfully synthesized in ethanol solvent by ultrasound method. The morphology, structural features and physicochemical properties of $\mathrm{CuCo}$ ZIFs were analyzed by several techniques including powder X-ray diffraction, thermo-gravimetric analysis, Fourier-transform infrared spectroscopy, energy-dispersive X-ray spectroscopy, scanning electron microscope, and nitrogen physisorption measurements. The results showed that CuCo-ZIFs nanocrystals demonstrated a ZIF-67-like polyhedral morphology with high thermal stability. The Brunauer-Emmett-Teller surface areas of CuCo-ZIFs were achieved approximately $1172 \mathrm{~m}^{2} / \mathrm{g}$, while its Langmuir surfaces areas were achieved approximately $1907 \mathrm{~m}^{2} / \mathrm{g}$.
\end{abstract}

\section{INTRODUCTION}

As a subclass of metal-organic frameworks (MOFs), zeolitic imidazolate frameworks (ZIFs) are a new type of porous crystals with extended three-dimensional structures constructed from tetrahedrally coordinated metal centers (typically $\mathrm{Zn}$ and $\mathrm{Co}$ ) bridged by imidazolate derivatives $\left(\mathrm{Im}^{-}\right.$) (Yao \& Wang, 2014). Specifically, the transition metal ion centers $(M=Z n$ and $C o)$ are attached to nitrogen atoms at the 1,3-positions of the imidazolate bridging ligand $\left(\mathrm{Im}=\mathrm{C}_{3} \mathrm{~N}_{2} \mathrm{H}_{3}{ }^{-}\right)$to form an angle of $145^{\circ}$ at the $\mathrm{M}-\mathrm{Im}-\mathrm{M}$ center similar to the $\mathrm{Si}-\mathrm{O}-\mathrm{Si}$ angle in silicas and zeolites (Tan et al., 2010). The unique properties of ZIFs such as high surface areas, excellent thermal and chemical stability help them to have been widely used for several potential applications including sensing (Lu \& Hupp, 2010), separation (Song et al., 2012), gas storage (Mu et al.,
2012), catalysis (Dey and Banerjee, 2013; Zhang et al., 2014) and adsorption (Li et al., 2016).

In recent years, bimetallic zeolitic imidazolate frameworks are being popularly studied. One of them must be mentioned is ZnCo-ZIFs which is composed of $\mathrm{Zn}^{2+}, \mathrm{Co}^{2+}$ cations and 2-methylimidazole anions with a sodalite-related structure (Han et al., 2019). According to a study on the adsorption capacity of $\mathrm{ZnCo}-\mathrm{ZIFs}$ to $\mathrm{CO}_{2}, \mathrm{CH}_{4}, \mathrm{~N}_{2}$ gases, it showed that ZnCo-ZIFs achieved higher efficiency than ZIF-8 and ZIF-67 due to the large specific surface areas and high porosity (Zhou et al., 2017). Similarly, bimetallic CuCo-ZIFs materials with a combination of both $\mathrm{Cu}^{2+}$ and $\mathrm{Co}^{2+}$ ions in the structural framework had more outstanding characteristics than monometallic ZIFs. In 2012, Yang et al. successfully studied and applied Cu-doped ZIF-67 materials (denoted: Cu/ZIF-67) in the field of gas 
adsorption and photocatalytic degradation of organic dyes (Yang et al., 2012). According to this study, $\mathrm{Cu} / \mathrm{ZIF}-67$ was synthesized under solvothermal conditions by mixing $\mathrm{Cu}(\mathrm{COO})_{2}, \mathrm{Co}(\mathrm{COO})_{2}$ and 2-methylimidazole in mixed ethyleneurea hemihydrate (e-urea) and ethanol solvent at $140^{\circ} \mathrm{C}$ for 7 days. However, it had low efficiency because of limited experimental conditions, complicated synthesis processes, and time-consuming. To overcome those drawbacks, Khan and Jhung (2015) reported that the use of high intensity ultrasonic waves was a versatile synthetic method for nanostructured materials. The ultrasonic waves could not only lead to homogeneous crystal formation but also speed up the reaction and decrease the synthesis time (Kim and Kim 2008; Suslick, 1990). Therefore, in this work, $\mathrm{CuCo}$-ZIFs were prepared by an ultrasound method in ethanol solvent at ambient temperature. Compared with organic solvents such as $N, N$-Dimethylformamide (DMF), methanol, acetone,... ethanol was considered a green solvent because it was biodegradable and less toxic. Besides, the synthesis process was carried out under mild conditions and shortened reaction times while the sample still had high crystallinity. The physicochemical properties of CuCo-ZIFs were then characterized by several techniques. This material has a great potential for applications in the catalytic fields.

\section{EXPERIMENT}

\subsection{Materials}

2-methylimidazole $\left(\mathrm{C}_{4} \mathrm{H}_{6} \mathrm{~N}_{2}, 99 \%\right)$ was purchased from Sigma-Aldrich. Cobalt (II) nitrate hexahydrate $\left(\mathrm{Co}\left(\mathrm{NO}_{3}\right)_{2} \cdot 6 \mathrm{H}_{2} \mathrm{O}, \geq 99 \%\right.$ purity), copper (II) nitrate trihydrate $\left(\mathrm{Cu}\left(\mathrm{NO}_{3}\right)_{2} .3 \mathrm{H}_{2} \mathrm{O}, \geq 99 \%\right.$ purity), ethanol $\left(\mathrm{C}_{2} \mathrm{H}_{5} \mathrm{OH}, \geq 99.5 \%\right.$ purity) were purchased from Xilong chemical Co., Ltd, China. All the reagents were used without further purification.

\subsection{Characterization techniques}

$\mathrm{X}$-ray powder diffraction (XRD) patterns were recorded using a $\mathrm{Cu} \mathrm{K} \alpha(\lambda=1.5406 \AA)$ radiation source on a D8 Advance - Brucker powder diffractometer. Energy-dispersive X-ray spectroscopy (EDX) was used to analyze elemental composition of $\mathrm{CuCo}-$ ZIFs. The morphological features were examined by scanning electron microscope (SEM, Hitach S4800). The specific surface areas of the samples were calculated using Brunauer-Emmett-Teller (BET) method. Fourier-transform infrared spectros- copy (FT-IR) were obtained on a Bruker TENSOR27 instrument, with samples being dispersed on potassium bromide pallets. Thermo-gravimetric analysis (TGA) was performed on a thermobalance TA instrument TGA-Q500 with the heating rate of $10^{\circ} \mathrm{C} / \mathrm{min}$ in the air atmosphere.

\subsection{Synthesis of CuCo-ZIFs}

CuCo-ZIFs were synthesized at ambient temperature in ethanol solvent. CuCo-ZIFs was synthesized according to the previous report with the molar ratio of $\mathrm{Co}^{2+}$ and $\mathrm{Cu}^{2+}$ is 1:1 (Ma et al., 2015). The molar ratio of metal salts and 2-MIm was fixed to 1:4 (Ma et al., 2015). Briefly, $\mathrm{Co}\left(\mathrm{NO}_{3}\right)_{2} \cdot 6 \mathrm{H}_{2} \mathrm{O}(0.582 \mathrm{~g}, 2$ $\mathrm{mmol})$ and $\mathrm{Cu}\left(\mathrm{NO}_{3}\right)_{2} .3 \mathrm{H}_{2} \mathrm{O}(0.483 \mathrm{~g}, 2 \mathrm{mmol})$ were separately dissolved in $10 \mathrm{~mL}$ ethanol. Another solution was prepared by dissolving 2-MIm $(1.3136 \mathrm{~g}$, $16 \mathrm{mmol}$ ) in $20 \mathrm{~mL}$ ethanol. Then, copper nitrate solution was slowly added to the cobalt nitrate solution. The mixture was stirred for $15 \mathrm{~min}$ to form a homogeneous mixture. After that, this mixture was dropped in 2-MIm solution under magnetic stirring, a purple suspension was formed. Subsequently, the mixture salts and 2-MIm were subjected to ultrasound for $15 \mathrm{~min}$ and maintained at ambient temperature for $24 \mathrm{~h}$. The purple precipitation was then obtained by centrifugation $(6,000 \mathrm{rpm}, 15 \mathrm{~min})$. The purple solid was washed by ethanol $(3 \times 10 \mathrm{~mL})$ for 4 days. Finally, the sample was dried at $60^{\circ} \mathrm{C}$ to obtain the CuCo-ZIFs crystals. The molar of $\mathrm{Co}^{2+}$ and $\mathrm{Cu}^{2+}$ were altered by varying the initial concentration of two metal salts $\left(\mathrm{Co}^{2+}: \mathrm{Cu}^{2+}\right.$ molar ratio $=1: 4$, $1: 8,1: 12,1: 16)$.

\section{RESULTS AND DISCUSSION}

\subsection{Characterization of the CuCo-ZIFs}

Powder X-ray diffraction (PXRD) patterns of the synthesized CuCo-ZIFs displayed sharp and clear peaks. Although five samples were synthesized in different ratios, PXRD results are almost the same (Figure 1). At the $\mathrm{Co}^{2+}: \mathrm{Cu}^{2+}$ molar ratios such as $1: 1$, $1: 4,1: 8,1: 12,1: 16$, the clearly peaks indexed to $2 \theta$ values of $7.4^{\circ}, 10.4^{\circ}, 12.8^{\circ}, 14.8^{\circ}, 16.5^{\circ}, 18.1^{\circ}, 24.5^{\circ}$, $25.6^{\circ}, 26.7^{\circ}, 29.7^{\circ}, 30.8^{\circ}$ and $31.5^{\circ}$ corresponding to (011), (002), (112), (022), (013), (222), (114), (233), (134), (044), (244) and (235), respectively having a good match with previously published results (Chen et al., 2020; Ma et al., 2015; Yang et al., 2012). Although those materials were synthesized in ethanol solvent with different molar ratios, they could completely create a crystal structure of CuCo-ZIFs. 


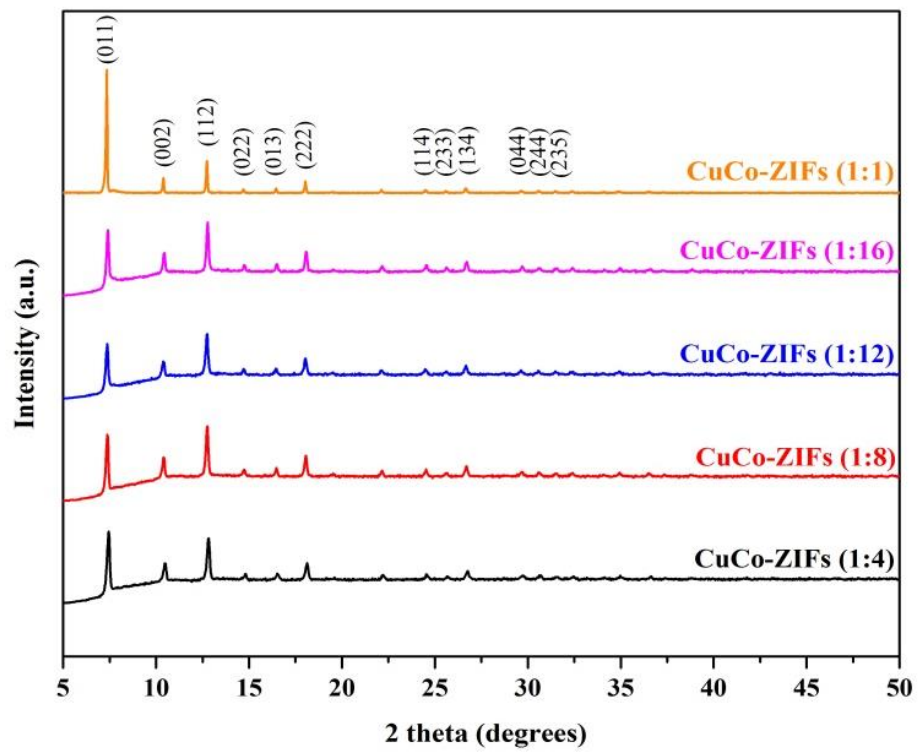

Figure 1. PXRD patterns of bimetallic CuCo-ZIFs samples with different molar ratios of cobalt nitrate and copper nitrate

However, the efficiency of the synthesis tended to decrease with increasing molar ratio of cobalt nitrate and copper nitrate (Table 1). Out of all the five ratios of CuCo-ZIFs synthesis, the ratio 1:1 had the highest efficiency $(15.9 \%)$ while the remaining ratios $(1: 4,1: 8,1: 12$ and 1:16) decreased slowly, as 13.2, $11.9,10.9$ and $9.3 \%$, respectively. This proved that the molar ratio of cobalt nitrate and copper nitrate

influenced the formation of bimetallic crystals. Therefore, the molar ratio 1: 1 was chosen to be the optimal condition for the next study.

Additionally, the element component was analyzed by energy-dispersive X-ray spectroscopy (EDX) (Figure 2). The EDX of CuCo-ZIFs demonstrated the full presence of elements in the compound including $\mathrm{C}, \mathrm{N}, \mathrm{Co}$ and $\mathrm{Cu}$.

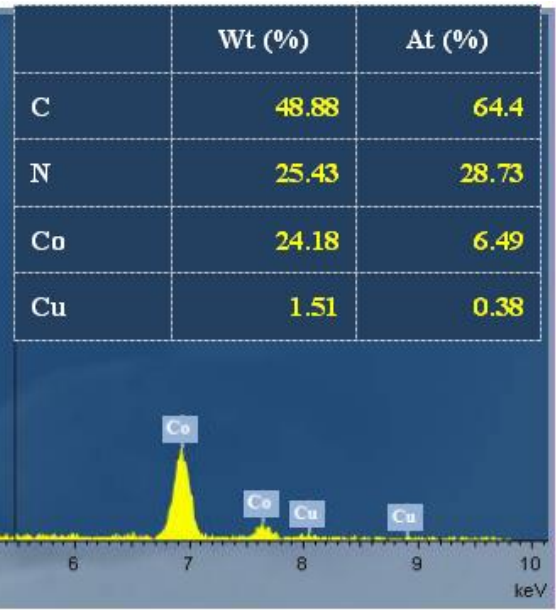

Figure 2. EDX spectrum of CuCo-ZIFs

To examine morphology of CuCo-ZIFs, a scanning electron microscope (SEM) was used. In detail, SEM micrographs of representative CuCo-ZIFs sample at the different magnification ratios were shown in Figure 3. The results demonstrated that the synthesized CuCo-ZIFs had relatively uniform cubic particles. This was consistent with the previous result of Gholinejad et al. (2020). 

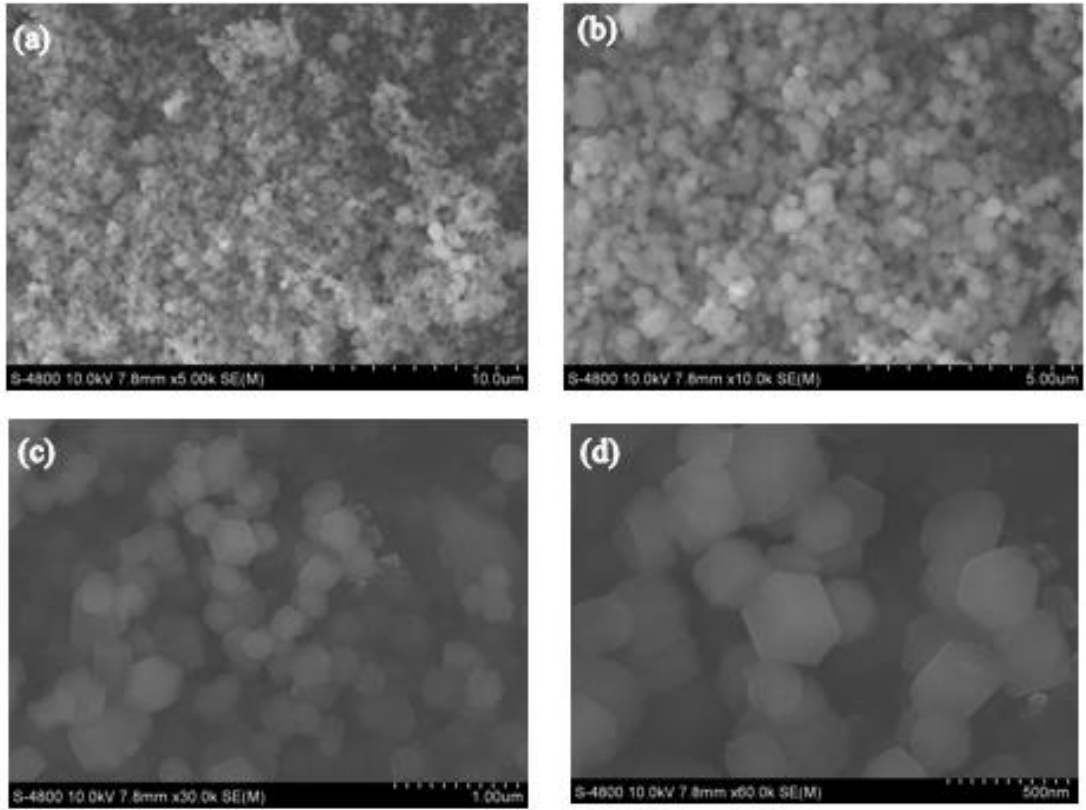

Figure 3. SEM micrographs of CuCo-ZIFs at the different magnification ratios (a) x5000, (b) x10000, (c) $\times 30000$; (d) $\times 60000$

Moreover, the Brunauer-Emmett-Teller surface areas of CuCo-ZIFs were achieved approximately $1172 \mathrm{~m}^{2} / \mathrm{g}$, while its Langmuir surfaces areas were achieved approximately $1907 \mathrm{~m}^{2} / \mathrm{g}$. Besides, according to the Dubinin-Astakhov method (DA), the pore size distribution of $\mathrm{CuCo}$-ZIFs was determined to be $9.6 \AA$ (Figure 4). It could be concluded that the obtained material had high surface areas. Compared to the previous report, the BET and Langmuir surface areas of $\mathrm{Cu} / \mathrm{ZIF}-67$ are $1324.1 \mathrm{~m}^{2} / \mathrm{g}$ and 1935.5 $\mathrm{m}^{2} / \mathrm{g}$ (Yang et al., 2012), which were a little higher than those of $\mathrm{CuCo}-\mathrm{ZIFs}$. However, the bimetallic CuCo-ZIFs material in this study were synthesised in a facile method include shortening time, saving energy and restricting environmental pollution.

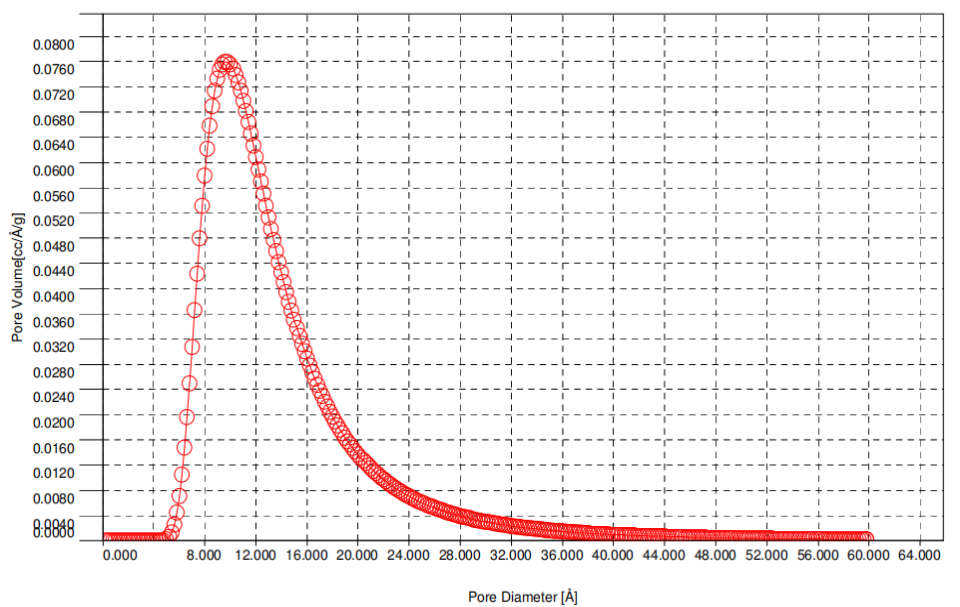

Figure 4. The pore size distributions of CuCo-ZIFs was analyzed by DA method

Figure 5 shows $\mathrm{N}_{2}$ adsorption isotherms of the CuCo-ZIFs sample. The result showed that the adsorbed amount increased suddenly at a high relative pressure and this phenomenon was similar to the work of Yang et al. (2012). It might be due to physisorbed liquid nitrogen on the crystal surfaces of the nanoparticles. 

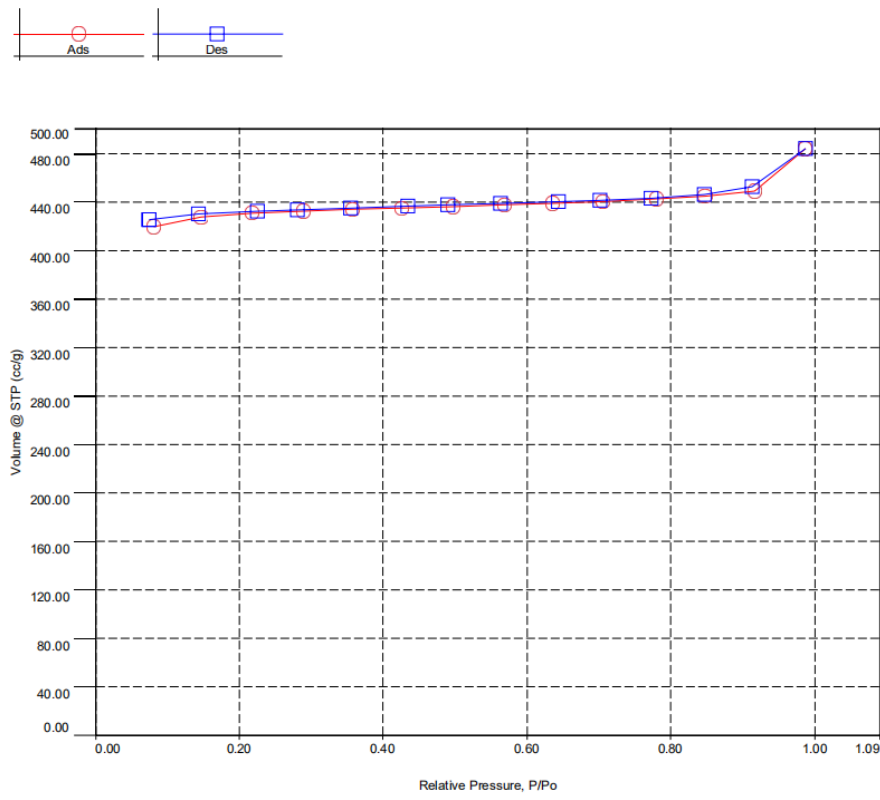

Figure 5. $\mathrm{N}_{2}$ adsorption/desorption isotherms of $\mathrm{CuCo}-\mathrm{ZIFs}$

$\mathrm{CuCo-ZIFs}$ were then measured by FT-IR to determine the presence of characteristic functional groups in the sample, and compared with FT-IR spectra of ZIF-67 and 2-MIm. Figure 6 showed that the spectrum of CuCo-ZIFs had many similarities with ZIF-67 but there were differences with 2-MIm. In the $600-1500 \mathrm{~cm}^{-1}$ region, the spectral shape of all three samples showed similar, although different peak intensity, because of imidazole ring oscillation. One vibration stretched $\mathrm{N}-\mathrm{H}$ at $1842.17 \mathrm{~cm}^{-1}$ in 2MIm and it disappeared for ZIF-67 and CuCo-ZIFs. This demonstrated the reduction of N-H groups in 2-MIm when combined with metal ions (Zhou et al.,
2017). Besides, the position $1595.36 \mathrm{~cm}^{-1}$ of $2-\mathrm{MIm}$ was the oscillation of $\mathrm{C}=\mathrm{N}$ bond corresponding to ZIF-67 and CuCo-ZIFs were $1585.18 \mathrm{~cm}^{-1}$ and $1582.40 \mathrm{~cm}^{-1}$, respectively. The peak at $2924.3 \mathrm{~cm}^{-1}$ appeared in CuCo-ZIFs and ZIF-67 could be attributed to the stretching mode of $\mathrm{C}-\mathrm{H}$ of the aliphatic chain and aromatic ring in 2-MIm. Notably, the position $421.36 \mathrm{~cm}^{-1}$ and $423.97 \mathrm{~cm}^{-1}$ correspondings to ZIF-67 and CuCo-ZIFs were the oscillation position of the metal-N bond. It means that the $\mathrm{CuCo-ZIFs} \mathrm{material} \mathrm{completely} \mathrm{achieved} \mathrm{the} \mathrm{de-}$ sired crystal structure (Gholinejad et al., 2020).

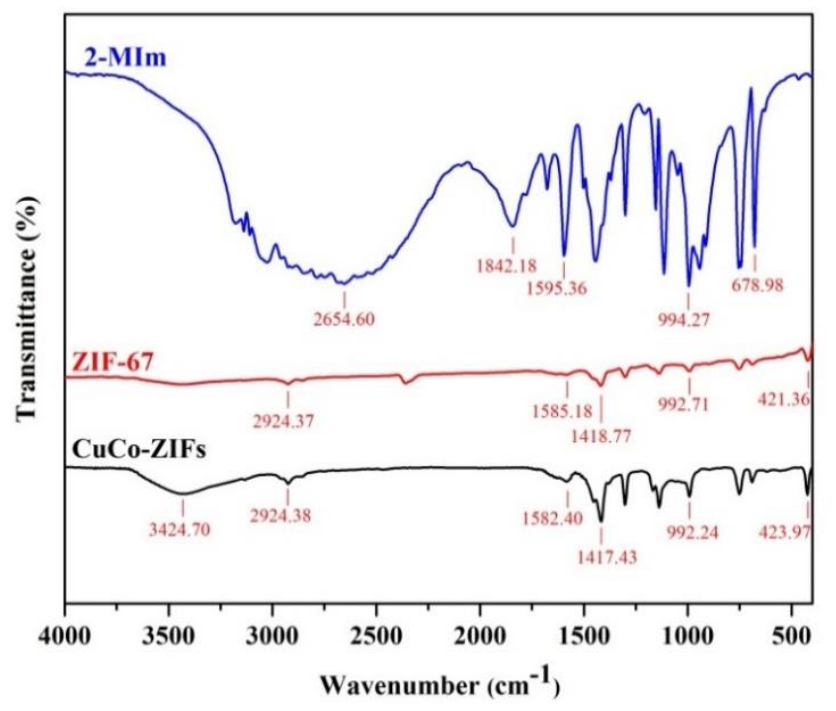

Figure 6. FT-IR spectra of 2-MIm, ZIF-67 and CuCo-ZIFs 
Thermal stability was one of the important criteria for solid materials. Hence, thermo-gravimetric analysis (TGA) was performed for CuCo-ZIFs in the temperature range from $30^{\circ} \mathrm{C}$ to $800^{\circ} \mathrm{C}$ (Figure 7). The results showed that CuCo-ZIFs had a stable structure over a wide temperature range. Specifically, the weight of the material decreased slightly by about $4 \%$ to $5 \%$ when the temperature was between $30^{\circ} \mathrm{C}$ and $510^{\circ} \mathrm{C}$. It was due to the evaporation of small-sized impurities, residual ethanol sol- vents or unreacted 2-MIm (Kaur et al., 2016). A pronounced weight loss of about $46 \%$ was observed from $510^{\circ} \mathrm{C}$ to $800^{\circ} \mathrm{C}$, this was the process of decomposition of $\mathrm{CuCo}$-ZIFs to form metal oxides with a residual mass of only about $48.9 \%$. Besides, the sample still tended to lose weight when the temperature exceeded $800^{\circ} \mathrm{C}$ as it continued to decompose until the structure was completely broken down. Generally, the TGA curve showed that the thermal stability of $\mathrm{CuCo}-\mathrm{ZIFs}$ was up to $510^{\circ} \mathrm{C}$ and be considered superior to ZIF-67 (Chen et al., 2014).

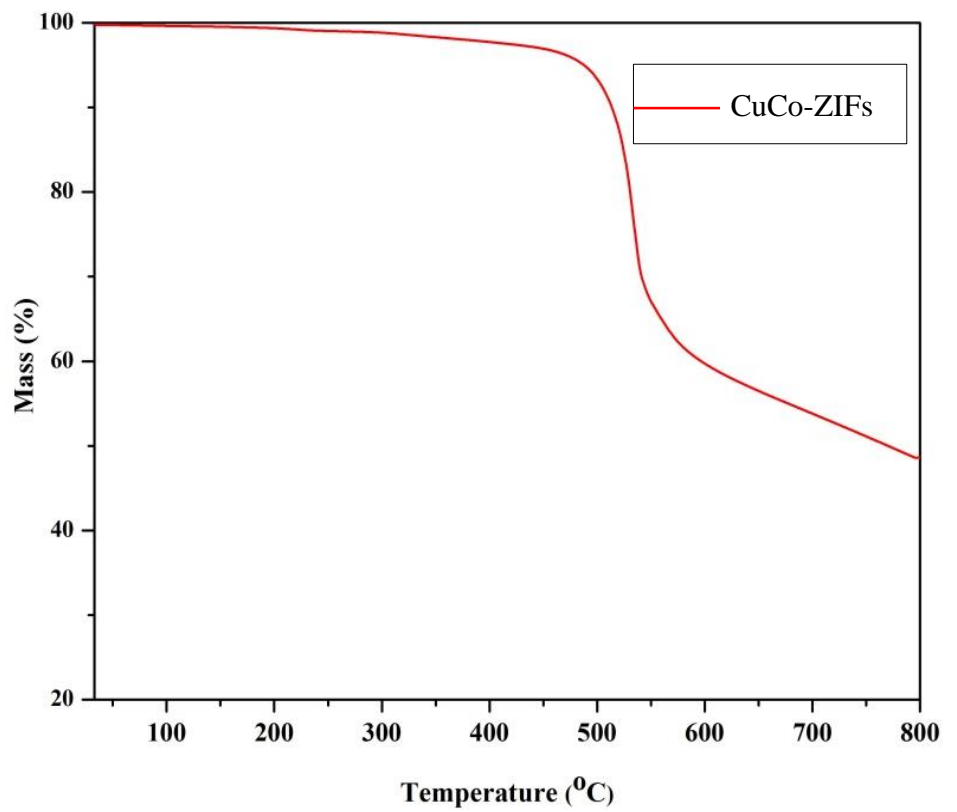

Figure 7. TGA curve of CuCo-ZIFs

\section{CONCLUSIONS}

In summary, CuCo-ZIFs has been successfully prepared in ethanol solvent by ultrasound method. It is a simple synthesis, low energy consumption and using environmentally friendly solvent compared to previous studies. Meanwhile, the material still has high crystal strength and polymorphic morphology, which is consistent with other studies. On one hand, the thermal stability of CuCo-ZIFs was higher than ZIF-67. Furthermore, this material has a high surface area which is an outstanding advantage when it is used as a catalyst. Thus, this study provides more new features in the study of catalytic materials belonging to the MOF group.

\section{ACKNOWLEDGMENTS}

This study is funded in part by the Can Tho University Improvement Project VN14-P6, supported by a Japanese ODA loan.

\section{REFERENCES}

Chen, E. X., Yang, H. \& Zhang, J. (2014). Zeolitic imidazolate framework as formaldehyde gas sensor. Inorganic Chemistry, 53(11), 5411-5413. https://doi.org/10.1021/ic500474j

Chen, W., Jia, Y., Yu, X., et al. (2020). Facile synthesis of bimetallic zeolite imidazolate framework with enhanced lithium storage performance. Ionics, 26(21), 1-9. https://doi.org/10.1007/s11581-019-03390-x

Dey, C., \& Banerjee, R. (2013). Controlled synthesis of a catalytically active hybrid metal-oxide incorporated zeolitic imidazolate framework (MOZIF). Chemical Communications, 49(59), 6617-6619. https://doi.org/10.1039/C3CC42904K

Gholinejad, M., Naghshbandi, Z. \& Sansano, J. M. (2020). Co/Cu bimetallic ZIF as new heterogeneous catalyst for reduction of nitroarenes and dyes. Applied Organometallic Chemistry, 34(4), 1-10. https://doi.org/10.1002/aoc.5522

Han, X., Ling, X., Wang, Y., et al. (2019). Generation of nanoparticle, atomic-czluster, and single-atom cobalt 
catalysts from zeolitic imidazole frameworks by spatial isolation and their use in zinc-air batteries. Angewandte Chemis, 131(16), 54135418. https://doi.org/10.1002/anie.201901109

Kaur, G., Rai, R. K., Tyagi, D., et al. (2016). Room-temperature synthesis of bimetallic $\mathrm{Co}-\mathrm{Zn}$ based zeolitic imidazolate frameworks in water for enhanced $\mathrm{CO}_{2}$ and $\mathrm{H}_{2}$ uptakes. Journal of Materials Chemistry A, 4(39), 14932-14938. https://doi.org/10.1039/C6TA04342A

Khan, N. A., \& Jhung, S. H. (2015). Synthesis of metalorganic frameworks (MOFs) with microwave or ultrasound: Rapid reaction, phase-selectivity, and size reduction. Coordination Chemistry Reviews, 285, 1123. https://doi.org/10.1016/j.ccr.2014.10.008

Kim, K. H., \& Kim, K. B. (2008). Ultrasound assisted synthesis of nano-sized lithium cobalt oxide. Ultrasonics Sonochemistry, 15(6), 1019-1025. https://doi.org/10.1016/j.ultsonch.2007.11.004

Li, Y., Zhou, K., He, M., \& Yao, J. (2016). Synthesis of ZIF-8 and ZIF-67 using mixed-base and their dye adsorption. Microporous and Mesoporous Materials, 234, 287-292. https://doi.org/10.1016/j.micromeso.2016.07.039

Lu, G., \& Hupp, J. T. (2010). Metal-Organic Frameworks as Sensors: A ZIF-8 Based Fabry-Pérot Device as a Selective Sensor for Chemical Vapors and Gases. Journal of the American Chemical Society, 132(23), 7832-7833. https://doi.org/10.1021/ja101415b

Ma, J., Wang, H., Yang, X., Chai, Y., \& Yuan, R. (2015). Porous carbon-coated $\mathrm{CuCo}_{2} \mathrm{O}_{4}$ concave polyhedrons derived from metal-organic frameworks as anodes for lithium-ion batteries. Journal of Materials Chemistry A, 3(22), 12038-12043. https://doi.org/10.1039/C5TA00890E

Mu, L., Liu, B., Liu, H., Yang, Y., Sun, C., \& Chen, G. (2012). A novel method to improve the gas storage capacity of ZIF-8. Journal of Materials Chemistry, 22(24), 12246-12252.

https://doi.org/10.1039/C2JM31541F
Song, Q., Nataraj, S. K., Roussenova, M. V., Tan, J. C., Hughes, D. J., Li, W., ... \& Sivaniah, E. (2012). Zeolitic imidazolate framework (ZIF-8) based polymer nanocomposite membranes for gas separation. Energy \& Environmental Science, 5(8), 8359-8369. https://doi.org/10.1039/C2EE21996D

Suslick, K. S. (1990). Sonochemistry. Science, 247(4949), 1439-1445. DOI: 10.1126/science.247.4949.1439

Tan, J. C., Bennett, T. D., \& Cheetham, A. K. (2010). Chemical structure, network topology, and porosity effects on the mechanical properties of Zeolitic Imidazolate Frameworks. Proceedings of The National Academy Of Sciences, 107(22), 9938-9943. https://doi.org/10.1073/pnas.1003205107

Yang, H., He, X. W., Wang, F., Kang, Y., \& Zhang, J. (2012). Doping copper into ZIF-67 for enhancing gas uptake capacity and visible-light-driven photocatalytic degradation of organic dye. Journal of Materials Chemistry, 22(41), 21849-21851. https://doi.org/10.1039/C2JM35602C

Yao, J., \& Wang, H. (2014). Zeolitic imidazolate framework composite membranes and thin films: Synthesis and applications. Chemical Society Reviews, 43(13), 4470-4493. https://doi.org/10.1039/C3CS60480B

Zhang, F., Wei, Y., Wu, X., Jiang, H., Wang, W., \& Li, H. (2014). Hollow Zeolitic Imidazolate Framework Nanospheres as Highly Efficient Cooperative Catalysts for [3+3] Cycloaddition Reactions. Journal of the American Chemical Society, 136(40), 1396313966. https://doi.org/10.1021/ja506372z

Zhou, K., Mousavi, B., Luo, Z., Phatanasri, S., Chaemchuen, S., \& Verpoort, F. (2017). Characterization and properties of $\mathrm{Zn} / \mathrm{Co}$ zeolitic imidazolate frameworks vs. ZIF-8 and ZIF-67. Journal of Materials Chemistry A, 5(3), 952-957. https://doi.org/10.1039/C6TA07860E 